\title{
Orbits of 10 interferometric binary systems calculated by using the improved Koval'skij method ${ }^{\star}$
}

\author{
D. Olević ${ }^{1,2}$ and Z. Cvetković ${ }^{1,2}$ \\ 1 Astronomical Observatory, Volgina 7, 11160-Belgrade 74, Serbia and Montenegro \\ 2 Institute Isaac Newton of Chile, Yugoslavia Branch
}

Received 11 July 2003 / Accepted 4 October 2003

\begin{abstract}
Orbital elements are presented of 10 interferometric binary systems with no previous orbit determination: WDS 07143-2621 (FIN 323), WDS 12064-6543 (FIN 367Aa), WDS 12446-5717 (FIN 65), WDS 13320-6519 (FIN 369), WDS 13574-6229 (FIN 370), WDS 14189+5452 (CHR 137), WDS 14373-4608 (FIN 318 Aa), WDS 16115+0943 (FIN 354), WDS 17018-5108 (I 1306), WDS 17221-7007 (FIN 373). The method used (Koval'skij-Olević) is briefly described.
\end{abstract}

Key words. techniques: interferometric - stars: binaries: close

\section{Introduction}

Even though a large number of double and multiple systems has been discovered to date (WDS, Mason et al. 2003 contains over 84000 systems), there is sufficient data for only a few thousand systems for the Keplerian motion to be clearly discerned.

The Sixth Catalog of Orbits of Visual Binary Stars (Hartkopf \& Mason 2003) contains about 1700 pairs.

By the use of large-aperture telescopes and high resolution techniques (speckle interferometry, photo-electric occultation timing etc.) more precise data are supplied allowing the calculation of preliminary orbital elements from shorter observed arcs. Thereby the sample usable for various statistical analyses is enlarged such as: calculation of dynamical parallaxes and masses, studies of dynamical systems evolution etc.

From the Fourth Catalog of Interferometric Measurements of Binary Stars (Hartkopf et al. 2003) we selected 10 pairs exhibiting significant changes in position angles and separations, observed eight or more times. The systems concerned are: WDS 07143-2621 (FIN 323) = HIP 34981, WDS 12064 $6543($ FIN $367 \mathrm{Aa})=$ HIP 59050, WDS 12446-5717 $($ FIN 65 $)=$ HIP 62179, WDS 13320-6519 (FIN 369) = HIP 66005, WDS 13574-6229 (FIN 370) = HIP 68170, WDS 14189+5452 $($ CHR 137) $=$ HIP 69958, WDS 14373-4608 (FIN 318 Aa $)=$ HIP 71500, WDS 16115+0943 (FIN 354) = HIP 79337, WDS 17018-5108 (I 1306) = HIP 83321, WDS 17221-7007 $($ FIN 373$)=$ HIP 84979 .

There is a small number of optical observations of these pairs of minor precision.

\footnotetext{
Send offprint requests to: D. Olević, e-mail: dolevic@aob.bg.ac.yu

* Table 2 is only available in electronic form at http://www.edpsciences.org
}

\section{Method of orbit calculation}

The best known method of orbital element calculation is that of Thiele-van den Bos (Thiele 1883; Dommanget 1954; van den Bos 1926, 1932) developed by perfecting Thiele's method. It requires the knowledge of three normal places and an area constant $\left(t_{i}, \theta_{i}, \rho_{i} ; 2 C\right), i=1,2,3$.

In recent years two analytical methods of orbit determination have been proposed by Docobo (1985), Eichhorn (1985) and Eichhorn \& Xu (1990).

One of the chief improvements of the method proposed by Docobo consists in its not requiring the knowledge of the area constant. The method is based on a mapping from the interval $(0,2 \pi)$ into the family of Keplerian orbits whose apparent orbits pass through three base points (normal places of high weight). In reality, that of Thiele-Innes-van den Bos is a particular case of this method.

CHARA developed an algorithm (Hartkopf et al. 1989; Mason et al. 1999) requiring preliminary knowledge of the period $P$, epoch of periastron passage $T$ and eccentricity $e$. The orbits are calculated according to the "three-dimensional adaptive grid search technique".

Koval'skij proposed as early as 1872 an analytical method, based on determination of five geometrical orbital elements $(\Omega, i, \omega, e$ and elipse parameter $p)$ from five coefficients $A_{k}$ $(k=1,2,3,4,5)$ of the conic Eq. (1a) (below). Two dynamical elements, the period $P$ and the epoch of periastron passage $T$, are obtained from the Keplerian Eq. (2). The method is described in detail in Subbotin (1968).

$$
\begin{aligned}
F(\mathbf{A}, \mathbf{x})= & A_{1} x_{i}^{2}+A_{2} y_{i}^{2}+2 A_{3} x_{i} y_{i} \\
& +2 A_{4} x_{i}+2 A_{5} y_{i}-1=0
\end{aligned}
$$


where $x_{i}=\rho_{i} \cos \theta_{i}$ and $y_{i}=\rho_{i} \sin \theta_{i} ;(\theta, \rho)$ - polar coordinates of $\mathrm{B}$ component with respect to $\mathrm{A}$ in the system, for epoch $t_{i}$ $(i=1,2, \ldots, n)$.

$\frac{2 \pi}{P}\left(t_{i}-T\right)=M\left(\theta_{i}, \Omega, \omega, i, e, a\right)$

where $M$ - mean anomaly and $a$ - semimajor axis of ellipse.

To obtain acceptable orbital elements by this method the observational data should cover the entire orbit within at least one period. Failing this, it often happens that real elliptic solution cannot be obtained. However, even with the elliptic solution arrived at, it can be used only as a first approximation (Eichhorn 1985, 1990; Eichhorn \& Xu 1990).

In order to enable this method to be applied even in cases where the observations cover a shorter arc of the orbit or there are gaps Olević introduced an improvement by using supplementary, fictive, observations. We named this method Koval'skij-Olević (KOVOLE).

The method has not been published in its entirety so far, so we present it here briefly.

We extend the polynomial (1a) with the system (1b) based on $f$ fictive observations $\left(\theta_{l}^{\prime}, \rho_{l}^{\prime}\right), l=1,2, \ldots, f$.

$$
\begin{aligned}
F^{\prime}\left(\mathbf{A}, \mathbf{x}^{\prime}\right)= & A_{1} x_{l}^{\prime 2}+A_{2} y_{l}^{\prime 2}+2 A_{3} x_{l}^{\prime} y_{l}^{\prime} \\
& +2 A_{4} x_{l}^{\prime}+2 A_{5} y_{l}^{\prime}-1=0
\end{aligned}
$$

where $x_{l}^{\prime}=\rho_{l}^{\prime} \cos \theta_{l}^{\prime}, y_{l}^{\prime}=\rho_{l}^{\prime} \sin \theta_{l}^{\prime}$.

The fictive position angles $\theta_{l}^{\prime}$ are uniformly distributed along the part of the arc not covered with observations. Since second order centred curves exclusively are under consideration, the angles $\theta_{l}^{\prime}$ will always be realized with the observations.

For radius-vectors $\rho_{l}^{\prime}$ are determined intervals $s_{l}=\left(\rho_{l_{\max }^{\prime}}^{\prime}-\right.$ $\left.\rho_{l_{\min }}^{\prime}\right)$ through which the orbit will certainly pass satisfying optimally the real observations along the $\operatorname{arc} L$. These intervals can easily and accurately be determined provided sufficiently wide limits are taken.

Let $\rho_{l}^{\prime}$ take, in the interval $s_{l}$, discrete values with the step $q$, e.g. $q \leq 00^{\prime \prime} 001$.

Then the system $[1(a)+1(b)]$ yields:

$S=\prod_{l=1}^{f} s_{l} / q$

sets of solutions for coefficients $A_{k}$.

The a priori weights $w_{i}$ of the equations of conditions (1a) can be determined, e.g. in the way proposed by Mason et al. (1999). The equations of conditions (1b) are of equal weight $G$. This quantity is determined by Eq. (3), which enables the system (1b) to be of equal weight as the system (1a). This is of particular importance as it ensures the coherence of the real and fictitious measurements.

$G=G\left(w_{i}, L, f\right)=\left(\sum_{1}^{n} w_{i}(360-L)\right) / L f$.

For each one of $S$ combinations one calculates the invariants $U_{1}, U_{2}$ and $U_{3}$ defined by matrices:

$U_{1}=\left[A_{1}+A_{2}\right]$,
$U_{2}=\left[\begin{array}{ll}A_{1} & A_{3} \\ A_{3} & A_{2}\end{array}\right]$

$U_{3}=\left[\begin{array}{ccc}A_{1} & A_{3} & A_{4} \\ A_{3} & A_{2} & A_{5} \\ A_{4} & A_{5} & 1\end{array}\right]$.

In cases where the conditions are simultaneously satisfied:

$\operatorname{det} U_{2}>0$ and $\left(\operatorname{det} U_{3} / \operatorname{det} U_{1}\right)<0$

the solution yields a real ellipse (Korn \& Korn 1961).

From the coefficients $A_{k}$ satisfying the above conditions one calculates the geometrical orbital elements $\Omega, \omega, i, e$ and $a$, and by applying the Keplerian Eq. (2) dynamical elements are derived: the period $P$ and the epoch of periastron passage $T$.

The best solution is the one yielding $D=D_{\min }$, the function $D$ being defined by:

$D=\left(\sum_{1}^{n} w_{i}\left[\left(x_{i}-x_{c_{i}}\right)^{2}+\left(y_{i}-y_{c_{i}}\right)^{2}\right]\right) / \sum_{1}^{n} w_{i}$

where $x_{i}, y_{i}$ are the observed rectangular coordinates and $x_{c_{i}}$, $y_{c_{i}}$ are calculated rectangular coordinates.

From the corresponding covariation matrices the formal errors of orbital elements are derived.

By this method a better accordance is established between the geometrical parameters of the conic (Kepler's first law) and the dynamical parameters of the Keplerian equation which implies the area law (Kepler's second law).

Olević has made use of this method's idea since 1985 (Ćatović \& Olević 1992). The method was complemented, being completely realized and tested only after a computer of adequate speed was put at our disposal. The suitable program has been designed by the author's collaborators P. Jovanović and Z. Cvetković.

\section{Results}

The orbital elements (equinox J2000) and the corresponding errors are listed in Table 1. These elements have been obtained from the interferometric measurements alone.

Table 2 contains the observational data used and their residuals. Asterisks (*) mark observations subjected to a change of quadrant. Double asterisks $(* *)$ indicate the measurements not used in the orbit calculation. The sign \# indicate the optical measurements. The ephemeride values are given in brackets.

The ephemerides for 2003-2007 are given in Table 3.

Plots 1-10 (Fig. 1) give the fitted orbit, the line of nodes, the observed interferometric positions (dotes) and their corresponding ephemeride positions (open circles). The optical measurements and the corresponding ephemeride positions are marked by squares.

\section{Notes on individual systems}

WDS 07143-2621 = Fin $323=$ Hip 34981. The measurements from 1952, 1953, 1954, 1977 and 1996 contain gross errors in the position angle $(\theta)$. These measurements have been ignored in the orbit calculation. The only optical measurement of 
Table 1. Orbital elements.

\begin{tabular}{cccccc}
\hline \hline Name & FIN 323 & FIN 367 Aa & FIN 65 & FIN 369 & FIN 370 \\
WDS & $07143-2621$ & $12064-6543$ & $12446-5717$ & $13320-6519$ & $13574-6229$ \\
HIP & 34981 & 59050 & 62179 & 66005 & 68170 \\
$m$ & $5.394-4.920$ & $6.788-7.142$ & $6.985-8.176$ & $7.358-7.540$ & $7.369-7.758$ \\
Sp. & B3IIIe & G8/K0III & A0IV/V & A8/A9IV & G3III/IV \\
\hline$P(\mathrm{yr})$ & $194.28 \pm 4.58$ & $103.18 \pm 5.81$ & $118.34 \pm 5.81$ & $40.44 \pm 0.91$ & $26.12 \pm 0.26$ \\
$T$ & $1972.81 \pm 4.52$ & $2055.22 \pm 1.94$ & $2065.09 \pm 0.81$ & $1994.64 \pm 0.49$ & $1967.02 \pm 0.43$ \\
$a\left(^{\prime \prime}\right)$ & $0.195 \pm 0.012$ & $0.152 \pm 0.005$ & $0.287 \pm 0.015$ & $0.192 \pm 0.026$ & $0.178 \pm 0.020$ \\
$e$ & $0.577 \pm 0.046$ & $0.299 \pm 0.011$ & $0.525 \pm 0.032$ & $0.194 \pm 0.058$ & $0.140 \pm 0.038$ \\
$i\left(^{\circ}\right)$ & $73.1 \pm 1.4$ & $146.2 \pm 1.4$ & $111.2 \pm 1.3$ & $57.0 \pm 3.0$ & $134.5 \pm 4.0$ \\
$\Omega\left(^{\circ}\right)$ & $151.4 \pm 0.8$ & $127.0 \pm 2.1$ & $61.8 \pm 0.9$ & $30.4 \pm 4.7$ & $105.0 \pm 4.8$ \\
$\omega\left(^{\circ}\right)$ & $50.6 \pm 4.1$ & $297.4 \pm 3.1$ & $113.7 \pm 4.8$ & $356.3 \pm 11.7$ & $148.0 \pm 13.4$ \\
\hline \hline Name & CHR 137 & FIN 318 Aa & FIN 354 & I 1306 & FIN 373 \\
WDS & $14189+5452$ & $14373-4608$ & $16115+0943$ & $17018-5108$ & $17221-7007$ \\
HIP & 69958 & 71500 & 79337 & 83321 & 84979 \\
$m$ & V:6.47 & $6.216-6.503$ & $7.193-7.515$ & $7.226-7.267$ & $5.897-6.456$ \\
Sp. & A5Vn & K0III & F0IV & A7V & B8/B9V \\
\hline$P(\mathrm{yr})$ & $13.39 \pm 0.77$ & $242.48 \pm 5.97$ & $48.12 \pm 5.62$ & $62.29 \pm 1.84$ & $51.44 \pm 2.66$ \\
$T$ & $1992.40 \pm 0.70$ & $2072.84 \pm 3.88$ & $1991.76 \pm 3.71$ & $2011.00 \pm 0.47$ & $1998.61 \pm 2.16$ \\
$a\left(^{\prime \prime}\right)$ & $0.090 \pm 0.010$ & $0.206 \pm 0.003$ & $0.142 \pm 0.050$ & $0.255 \pm 0.009$ & $0.105 \pm 0.008$ \\
$e$ & $0.704 \pm 0.122$ & $0.166 \pm 0.007$ & $0.021 \pm 0.020$ & $0.670 \pm 0.411$ & $0.252 \pm 0.084$ \\
$i\left(^{\circ}\right)$ & $112.1 \pm 3.3$ & $125.2 \pm 0.6$ & $92.1 \pm 3.9$ & $83.6 \pm 4.2$ & $47.1 \pm 10.0$ \\
$\Omega\left({ }^{\circ}\right)$ & $35.6 \pm 2.0$ & $113.7 \pm 0.9$ & $82.7 \pm 3.8$ & $19.5 \pm 1.5$ & $116.7 \pm 12.1$ \\
$\omega\left(^{\circ}\right)$ & $216.4 \pm 5.7$ & $256.8 \pm 4.3$ & $62.2 \pm 60.1$ & $107.7 \pm 21.9$ & $241.2 \pm 28.6$ \\
\hline & & & & & \\
\hline
\end{tabular}

Table 3. Ephemerides.

\begin{tabular}{|c|c|c|c|c|c|c|c|c|c|c|}
\hline \multirow{2}{*}{$\begin{array}{c}\text { WDS } \\
t\end{array}$} & \multicolumn{2}{|c|}{$07143-2621$} & \multicolumn{2}{|c|}{$12064-6543$} & \multicolumn{2}{|c|}{$12446-5717$} & \multicolumn{2}{|c|}{$13320-6519$} & \multicolumn{2}{|c|}{$13574-6229$} \\
\hline & $\theta$ & $\rho$ & $\theta$ & $\rho$ & $\theta$ & $\rho$ & $\theta$ & $\rho$ & $\theta$ & $\rho$ \\
\hline 2003.0 & 330.0 & .192 & 6.3 & .172 & 105.9 & .213 & 126.0 & .103 & 162.9 & .149 \\
\hline 2004.0 & 330.4 & .196 & 4.2 & .173 & 104.2 & .218 & 141.4 & .111 & 150.4 & .162 \\
\hline 2005.0 & 330.8 & .200 & 2.0 & .173 & 102.6 & .223 & 154.2 & .124 & 139.7 & .175 \\
\hline 2006.0 & 331.2 & .203 & 359.9 & .174 & 101.2 & .228 & 164.5 & .138 & 130.4 & .186 \\
\hline 2007.0 & 331.6 & .207 & 357.8 & .175 & 99.7 & .234 & 172.8 & .154 & 122.0 & .194 \\
\hline WDS & 14189 & 5452 & 14373 & 4608 & 16115 & 0943 & 17018 & 5108 & 17221 & 7007 \\
\hline$t$ & $\theta$ & $\rho$ & $\theta$ & $\rho$ & $\theta$ & $\rho$ & $\theta$ & $\rho$ & $\theta$ & $\rho$ \\
\hline 2003.0 & 353.2 & .059 & 323.0 & .180 & 264.0 & .122 & 17.1 & .224 & 56.9 & .062 \\
\hline 2004.0 & 324.4 & .035 & 321.9 & .181 & 263.6 & .130 & 17.8 & .212 & 68.9 & .069 \\
\hline 2005.0 & 254.5 & .029 & 320.8 & .182 & 263.3 & .137 & 18.5 & .197 & 78.7 & .076 \\
\hline 2006.0 & 154.6 & .013 & 319.7 & .184 & 263.0 & .141 & 19.4 & .178 & 86.9 & .083 \\
\hline 2007.0 & 45.4 & .063 & 318.7 & .185 & 262.8 & .143 & 20.5 & .153 & 93.8 & .090 \\
\hline
\end{tabular}



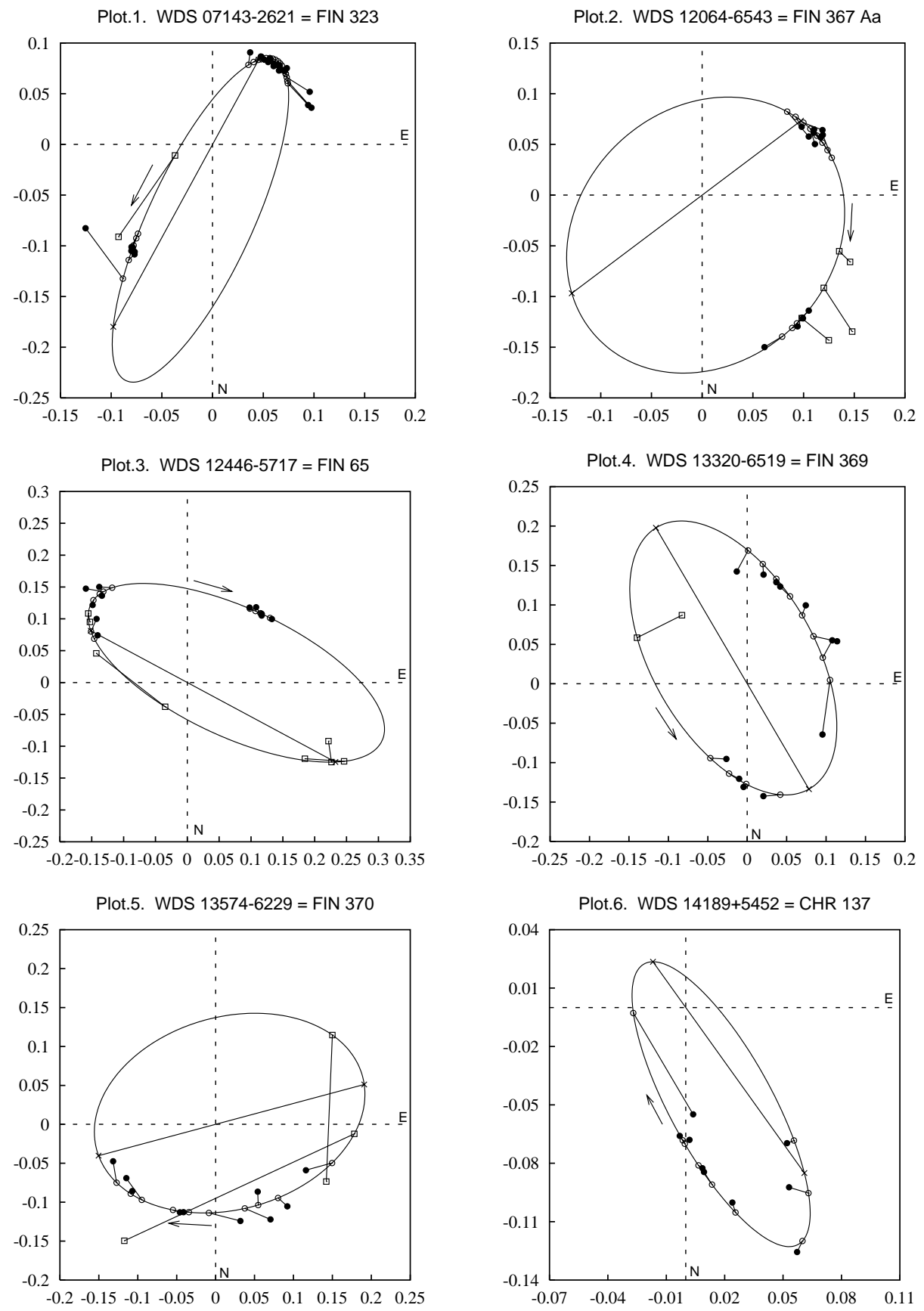

Fig. 1. Plots 1-10 (Fig. 1) give the fitted orbit, the line of nodes, the observed interferometric positions (dotes) and their corresponding ephemeride positions (open circles). The optical measurements and the corresponding ephemeride positions are marked by squares.

1980.230 is also ignored for the same reason. To achieve a better data fitting one had to change the quadrant either in Finsen's measurements or in those after 1977. We opted for the quadrant change.

WDS 12064-6543 = Fin 367 Aa $=$ Hip 59050. The three optical measurements lie close to the calculated ellipse pretty well.

WDS 12446-5717 = Fin $65=$ Hip 62179. The wrong quadrants in the measurements of 1991.25 (Hip) and 1993.093 (Hrt) are probably due to a lapse of the magnitude difference of 1.2. Of the four optical measurements only that of 1945.12 contains a large error.
WDS 13320-6519 = Fin $369=$ Hip 66005. The normal place formed for the instant 1960.57 , including also the measurement made on the occasion of its discovery, has a large error and was omitted from the orbit calculation. The single optical measurement of 1983.177 is imprecise.

WDS 13574-6229 = Fin $370=$ Hip 68170. Since its discovery (1960) the pair completed its whole period $(P=$ $26.125 \mathrm{yr}$ ). Both optical measurements contain large errors in the position angle.

WDS 14189+5452 = CHR $137=$ Hip 69958. This short period system $(P=13.392 \mathrm{yr}, a=0$ ’.090) contains a large error in the interferometric measurement of 1991.3271. 


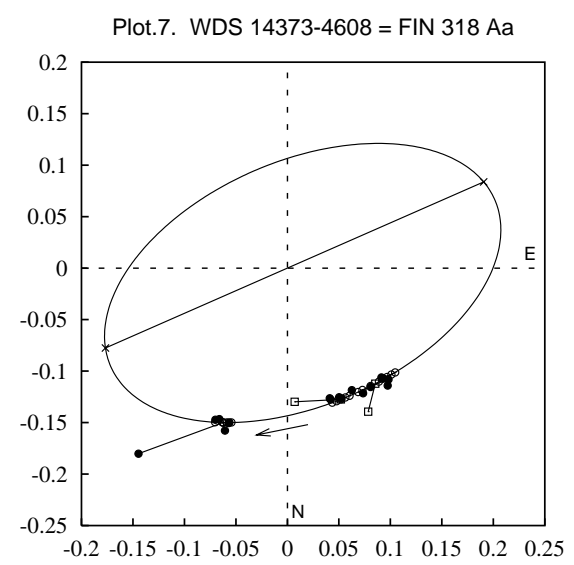

Plot.9. WDS 17018-5108 $=\mid 1306$

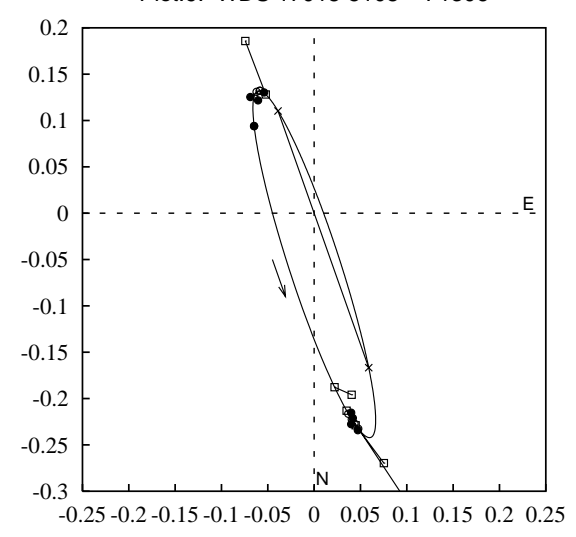

Fig. 1. continued.

WDS 14373-4608 = Fin 318 Aa $=$ Hip 71500. The components of this triple system are denoted A, a and B in the WDS catalog and as A, B and C in the Hipparcos catalog. The component $\mathrm{C}$ has a Hip 71502 entry. The system has two optical measurements, one of them from 1964.61 containing a considerable error in the position angle.

WDS 16115+0943 = Fin $354=$ Hip 79337. For the first four normal places formed by the discoverer (Finsen) from the observations made between 1959 and 1962, the quadrants have been changed. In 1963-1966 he made four separate measurements, failing, however, to measure the position angle. According to our calculation this pair with a $48 \mathrm{yr}$ period and $92^{\circ}$ inclination almost completed a whole revolution in the period concerned. The three optical measurements contain small errors; for the measurements of 1960.544 and 1961.59 the quadrants have been changed.

WDS 17018-5108 = I $1306=$ Hip 83321. In five attempts in the interval 1960-1965 Finsen failed to measure the position angle $(\theta)$, while the separation could only be estimated. The ephemerides show that in the time interval concerned $\rho \leq 0$ ". 1 which gives the pair's apparent magnitude (6.4-6.5), rendered precise measurements difficult. In three (1926.51, 1929.32 and 1985.33) out of four optical measurements the quadrant was changed. The accuracy is within the limits expected with this kind of measurement.

WDS 17221-7007 = Fin $373=$ Hip 84979. This, according to our calculated short-period system $(P=51.441 \mathrm{yr})$, has no optical measurements.

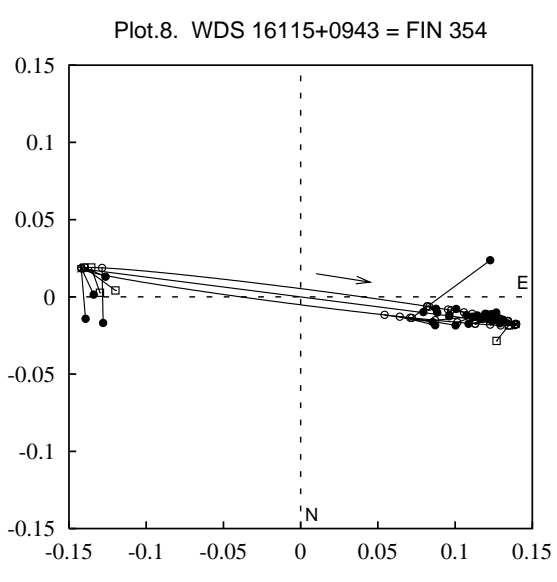

Plot.10. WDS $17221-7007=$ FIN 373

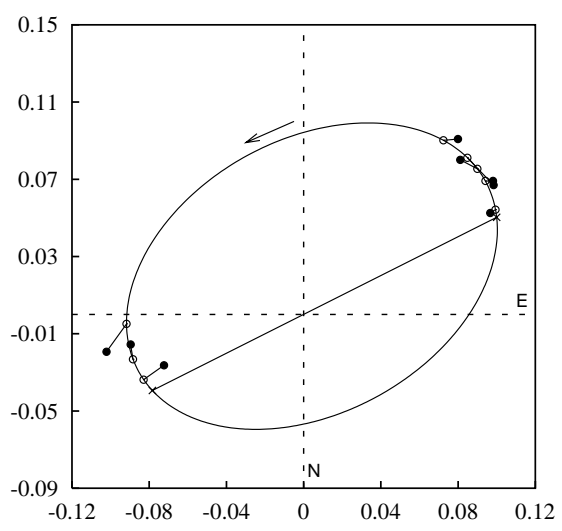

Gaps in the observations, easily seen in the plots for the Finsen pairs, discovered at the South African Astronomical Observatory $(\varphi=-26.2)$ appeared after the discoverer's death. In the southern hemisphere, until recently, the number of observations has been insufficient to continue the study of the motion of systems with high negative declinations.

\section{Conclusions}

In spite of difficulties met in the calculation of the orbital elements of the selected pairs, such as short arcs covered with observations, large gaps between two consecutive groups of observations and high inclinations, we obtained elements with small relative errors. Contributing to this was, on the one hand, enhanced accuracy of measurements thanks to the use of new techniques and, on the other, the KOVOLE method employed.

The obtained orbital elements are preliminary ones.

In view of the relatively short periods these systems should more often be included in interferometric observational programs. By so doing we might in the near future, obtain definite orbital elements. As these systems have their trigonometric parallaxes already determined by the Hipparcos mission, the sums of their masses may be determined. This is particularly important because the greater part (seven) of these systems, according to their spectral type, belong to the main sequence.

Acknowledgements. The authors express their gratitude to J. A. Docobo for useful remarks and suggestions. This work is a part of the 
project No. 1221 "Investigation of Double and Multiple Stars" supported by the Ministry of Science Technology and Development of Serbia.

\section{References}

Ćatović, Z., \& Olević, D. 1992, IAU Coll., 135, ASP Conf. Ser., 32, 217

Docobo, J. A. 1985, Cel. Mech., 46, 143

Dommanget, J. 1954, Observatoire Royal de Belgique, Astr. Bull., vol. IV, 8

Eichhorn, K. H. 1985, Ap\&SS, 110, 119

Eichhorn, K. H., \& Xu, Yu-Lin 1990, AJ, 358, 575

Hartkopf, W. I., McAlister, H. A., \& Franz, O. G. 1989, AJ, 98, 1014

Hartkopf, W. I., \& Mason, B. D. 2003, Sixth Catalog of Orbits of Visual Binary Stars, US Naval Observatory, Washington, http://ad.usno.navy.mil/wds/orb6.html
Hartkopf, W. I., Mason, B. D., Wycoff, G. L., \& McAlister, H. A. 2003, Fourth Catalog of Interferometric Measurements of Binary Stars, US Naval Observatory, Washington and Georgia State University, http://ad.usno.navy.mil/wds/int4.html

Korn, A. G., \& Korn, M. T. 1961, Mathematical Handbook for Scientists and Engineers (New York, Toronto, London: Mc. GrawHill Book Company, Inc.)

Mason, B. D., Douglas, G. G., \& Hartkopf, W. I. 1999, AJ, 117, 1023 Mason, B. D., Wycoff, G. L., \& Hartkopf, W. I. 2003, The Washington Visual Double Star Catalogue, US Naval Observatory, Washington, http://ad.usno.navy . mil/wds/wds.html

Subbotin, M. F. 1968, Vvedenie v teoreticheskuyu astronomiyu (Moskva: Nauka)

Thiele, T. N. 1883, Astron. Nachr., 104, 245

van den Bos, W. H. 1926, Union Circ., 68, 354

van den Bos, W. H. 1932, Union Circ., 86, 261 
D. Olević and Z. Cvetković: Orbits of 10 interferometric binary systems calculated by using Koval'skij method, Online Material $p 1$

\section{Online Material}


D. Olević and Z. Cvetković: Orbits of 10 interferometric binary systems calculated by using Koval'skij method, Online Material $p 2$

Table 2. Observations and residuals.

\begin{tabular}{ccrllrr}
\hline \hline \multicolumn{7}{c}{ WDS 07143-2621 $=$ FIN 323 } \\
\hline$t$ & $\theta$ & $\rho$ & $n$ & Obs. & $\Delta \theta$ & $\Delta \rho$ \\
\hline $1952.5000^{* *}$ & 112.1 & .102 & 7 & Fin & -16.7 & .006 \\
$1953.2300^{* *}$ & 110.1 & .104 & 7 & Fin & -20.0 & .007 \\
$1954.2300^{* *}$ & 118.1 & .109 & 2 & Fin & -13.7 & .010 \\
1955.2600 & 137.1 & .100 & 9 & Fin & 3.4 & .000 \\
1955.3100 & 135.5 & .102 & 1 & Fin & 1.7 & .002 \\
1956.2100 & 135.5 & .105 & 8 & Fin & 0.2 & .004 \\
1958.2400 & 139.6 & .102 & 5 & Fin & 1.1 & -.001 \\
1959.2500 & 140.8 & .101 & 5 & Fin & 0.7 & -.003 \\
1960.2400 & 137.8 & .098 & 6 & Fin & -3.8 & -.006 \\
1961.2900 & 141.8 & .098 & 5 & Fin & -1.4 & -.006 \\
1962.2700 & 142.7 & .100 & 5 & Fin & -2.1 & -.003 \\
1963.2460 & 145.9 & .102 & 5 & Fin & -0.4 & .000 \\
1964.2830 & 148.4 & .098 & 8 & Fin & 0.4 & -.002 \\
1965.2730 & 145.8 & .098 & 5 & Fin & -3.9 & .000 \\
1966.2780 & 150.5 & .099 & 4 & Fin & -1.0 & .004 \\
1967.2820 & 151.0 & .099 & 8 & Fin & -2.4 & .008 \\
1968.3210 & 157.6 & .098 & 6 & Fin & 2.0 & .012 \\
$1980.2300^{\circ *}$ & 134.4 & .13 & - & Wor & 28.1 & .091 \\
$1989.9336^{*}$ & 142.0 & .129 & 1 & Hrt & 1.1 & .010 \\
$1990.9137^{*}$ & 142.6 & .132 & 1 & Hrt & 0.7 & .006 \\
$1991.2500^{*}$ & 144.0 & .131 & 1 & Hip & 1.7 & .002 \\
$1993.0953^{*}$ & 144.6 & .133 & 1 & Hrt & 0.6 & -.008 \\
$1996.1752^{*}$ & 123.4 & .150 & 1 & Msn & -22.9 & -.009 \\
\hline
\end{tabular}

Table 2. continued.

\begin{tabular}{crrrlrr}
\hline \hline \multicolumn{7}{c}{ WDS 12064-6543 $=$ FIN 367 Aa } \\
\hline$t$ & $\theta$ & $\rho$ & $n$ & Obs. & $\Delta \theta$ & $\Delta \rho$ \\
\hline 1960.5100 & 118.8 & .120 & 3 & Fin & -15.1 & .003 \\
1961.5000 & 124.5 & .119 & 3 & Fin & -4.9 & -.001 \\
1962.5000 & 118.4 & .135 & 3 & Fin & -6.7 & .012 \\
1963.4700 & 114.4 & .122 & 2 & Fin & -6.7 & -.003 \\
1964.4830 & 115.9 & .130 & 4 & Fin & -1.1 & .003 \\
1965.4940 & 119.2 & .126 & 3 & Fin & 6.1 & -.004 \\
1966.4830 & 120.4 & .128 & 3 & Fin & 11.0 & -.003 \\
1967.4880 & 116.5 & .133 & 3 & Fin & 10.8 & .000 \\
$1979.2000^{\circ}$ & 65.7 & .16 & - & hz & -0.8 & .014 \\
$1984.3800^{\circ}$ & 47.7 & .20 & - & hz & -3.9 & .049 \\
1989.3033 & 42.7 & .155 & 1 & McA & 3.6 & -.001 \\
$1989.3900^{\circ}$ & 41.1 & .19 & - & hz & 3.1 & .034 \\
1990.3491 & 39.3 & .157 & 1 & Hrt & 2.9 & .000 \\
1991.2500 & 36.0 & .140 & 1 & Hip & 2.0 & .002 \\
1993.0930 & 22.3 & .162 & 1 & Hrt & -7.1 & .002 \\
\hline
\end{tabular}

Table 2. continued.

\begin{tabular}{cccllrr}
\hline \hline \multicolumn{7}{c}{ WDS $12446-5717=$ FIN 65 } \\
\hline \multicolumn{1}{c}{$t$} & $\theta$ & $\rho$ & $n$ & Obs. & $\Delta \theta$ & $\Delta \rho$ \\
\hline $1928.4300^{\diamond *}$ & 237.2 & .22 & - & Fin & -6.3 & -.055 \\
$1930.4800^{\diamond *}$ & 247.6 & .24 & - & $J$ & 6.3 & -.018 \\
$1945.1200^{\circ}$ & 252.3 & .15 & 2 & Vou & -65.4 & .098 \\
1952.4700 & 242.3 & .159 & 2 & Fin & -2.9 & -.002 \\
1953.4700 & 235.0 & .174 & 3 & Fin & -7.4 & .003 \\
$1956.4100^{\circ}$ & 238.2 & .18 & - & B & 3.0 & -.009 \\
1959.5000 & 230.8 & .192 & 2 & Fin & 1.7 & -.004 \\
1961.5100 & $: 224.6$ & $: .191$ & 3 & Fin & -0.4 & -.004 \\
1962.5000 & 227.3 & .217 & 1 & Fin & 4.3 & .023 \\
1964.5260 & 222.7 & .204 & 3 & Fin & 3.9 & .014 \\
1989.3033 & 140.4 & .153 & 1 & McA & 0.6 & .001 \\
1990.3463 & 137.6 & .160 & 1 & Hrt & 1.2 & .005 \\
$1991.2500^{*}$ & 312.0 & .157 & 1 & Hip & -1.5 & -.001 \\
1991.3894 & 132.6 & .159 & 2 & Hrt & -0.5 & .000 \\
$1993.0930^{*}$ & 306.9 & .166 & 1 & Hrt & -1.1 & .001 \\
\hline
\end{tabular}

Table 2. continued.

\begin{tabular}{crrllrr}
\hline \hline \multicolumn{7}{c}{ WDS 13320-6519 FIN 369 } \\
\hline \multicolumn{1}{c}{$t$} & $\theta$ & $\rho$ & $n$ & Obs. & $\Delta \theta$ & $\Delta \rho$ \\
\hline $1960.5700^{* *}$ & 56.2 & .115 & 3 & Fin & -36.9 & .010 \\
1961.5600 & $: 117.3$ & $: .121$ & 2 & Fin & 7.7 & .020 \\
1962.5400 & 115.5 & .126 & 5 & Fin & -10.6 & .023 \\
1963.5590 & 143.5 & .124 & 4 & Fin & 1.7 & .013 \\
1964.5430 & 161.4 & .130 & 7 & Fin & 7.0 & .007 \\
1965.5630 & 164.2 & .134 & 4 & Fin & -0.7 & -.004 \\
1966.5390 & 171.7 & .140 & 3 & Fin & -1.3 & -.013 \\
1967.5800 & 185.5 & .143 & 2 & Fin & 5.4 & -.026 \\
$1983.1770^{\circ}$ & 223.7 & .12 & 2 & Wor & -23.7 & -.031 \\
1989.3036 & 344.5 & .099 & 1 & McA & 10.8 & -.006 \\
1990.3437 & 355.2 & .121 & 1 & Hrt & 6.5 & .005 \\
1991.2500 & 358.0 & .131 & 1 & Hip & -1.5 & .004 \\
1993.0984 & 8.2 & .144 & 1 & Hrt & -8.5 & -.003 \\
\hline
\end{tabular}

Table 2. continued.

\begin{tabular}{crrllrr}
\hline \hline \multicolumn{7}{c}{ WDS $13574-6229=$ FIN 370 } \\
\hline$t$ & $\theta$ & $\rho$ & $n$ & Obs. & $\Delta \theta$ & $\Delta \rho$ \\
\hline 1960.5700 & 63.3 & .130 & 3 & Fin & -8.0 & -.028 \\
1962.5800 & 41.4 & .140 & 3 & Fin & 1.4 & .016 \\
1963.5610 & 30.2 & .141 & 5 & Fin & 11.3 & .027 \\
1964.5620 & 14.6 & .128 & 6 & Fin & 19.2 & .014 \\
1965.5810 & 338.1 & .122 & 3 & Fin & 4.8 & -.001 \\
1966.5680 & 301.3 & .134 & 4 & Fin & -14.1 & -.002 \\
1967.5780 & 290.0 & .140 & 2 & Fin & -10.3 & -.008 \\
$1980.2260^{\circ}$ & 62.8 & .16 & 2 & Wor & -64.7 & -.029 \\
$1985.3400^{\circ}$ & 322.0 & .19 & - & hz & 235.9 & .011 \\
1989.3036 & 32.1 & .102 & 1 & McA & 4.4 & -.015 \\
1991.2500 & 340.0 & .120 & 1 & Hip & -3.0 & .002 \\
1993.0983 & 308.8 & .137 & 1 & Hrt & -0.4 & -.004 \\
\hline
\end{tabular}


D. Olević and Z. Cvetković: Orbits of 10 interferometric binary systems calculated by using Koval'skij method, Online Material $p 3$

Table 2. continued.

\begin{tabular}{crrllrr}
\hline \hline \multicolumn{7}{c}{ WDS 14189+5452 $=$ CHR 137 } \\
\hline \multicolumn{1}{c}{$\theta$} & $\theta$ & $\rho$ & $n$ & Obs. & $\Delta \theta$ & $\Delta \rho$ \\
\hline 1987.2698 & 13.5 & .103 & 1 & $M c A$ & -0.2 & -.005 \\
1988.1683 & 6.4 & .085 & 1 & $M c A$ & -2.1 & -.007 \\
1988.6653 & 6.0 & .083 & 1 & $M c A$ & 1.4 & .002 \\
1989.1564 & $: 357.4$ & $: .066$ & 1 & McA & -2.2 & -.004 \\
1989.2273 & 1.7 & .068 & 1 & McA & 2.9 & .000 \\
$1991.3271^{* *}$ & 4.0 & .055 & 1 & McA & 88.1 & .028 \\
1994.2214 & 36.8 & .087 & 1 & Hrt & -2.4 & -.001 \\
1995.2318 & 29.9 & .107 & 2 & Hrt & -3.6 & -.008 \\
1997.1320 & 24.5 & .138 & 1 & Hrt & -2.1 & .004 \\
\hline
\end{tabular}

Table 2. continued.

\begin{tabular}{crrllrr}
\hline \hline \multicolumn{7}{c}{ WDS 14373-4608 } \\
\hline \multicolumn{1}{c}{$\theta$} & \multicolumn{1}{c}{$\rho$} & $n$ & Obs 318 Aa & $\Delta \theta$ & $\Delta \rho$ \\
\hline 1951.5300 & 41.2 & .142 & 2 & Fin & -4.4 & -.003 \\
1952.4900 & 40.9 & .140 & 4 & Fin & -3.1 & -.004 \\
1953.5600 & 40.7 & .150 & 2 & Fin & -1.4 & .007 \\
1955.5900 & 41.2 & .142 & 1 & Fin & -0.9 & .002 \\
$1956.4900^{\circ}$ & 29.6 & .16 & - & B & -7.9 & .019 \\
1957.5900 & 42.4 & .146 & 1 & Fin & 4.6 & .005 \\
1959.5800 & 35.2 & .141 & 3 & Fin & 1.7 & -.001 \\
1960.5900 & 31.4 & .142 & 1 & Fin & 0.0 & .000 \\
1962.5900 & 28.0 & .134 & 4 & Fin & 0.9 & -.007 \\
1963.5890 & 22.7 & .137 & 4 & Fin & -2.2 & -.003 \\
1964.6040 & 18.3 & .134 & 5 & Fin & -4.3 & -.004 \\
$1964.6100^{\circ}$ & 3.3 & .13 & - & B & -19.1 & -.008 \\
1965.5980 & 22.0 & .135 & 4 & Fin & 1.6 & -.001 \\
1966.5860 & 18.2 & .133 & 2 & Fin & 0.2 & .000 \\
1989.3037 & 339.4 & .160 & 1 & McA & -0.9 & .000 \\
1990.3464 & $: 339.3$ & $: .161$ & 1 & Hrt & 0.7 & -.001 \\
1991.2500 & 339.0 & .169 & 1 & Hip & 1.9 & .006 \\
$1991.2500^{* *}$ & $: 321.3$ & $: .231$ & 1 & Fab & -16.0 & -.069 \\
1991.3895 & 335.8 & .161 & 1 & Hrt & -1.1 & -.002 \\
1993.0989 & 334.6 & .163 & 1 & Hrt & 0.4 & -.002 \\
\hline
\end{tabular}

Table 2. continued.

\begin{tabular}{|c|c|c|c|c|c|c|}
\hline \multicolumn{7}{|c|}{ WDS 16115+0943 = FIN 354} \\
\hline$t$ & $\theta$ & $\rho$ & $n$ & Obs. & $\Delta \theta$ & $\Delta \rho$ \\
\hline $1959.5800^{*}$ & 96.2 & .140 & 1 & Fin & 13.2 & -.003 \\
\hline $1959.6100^{*}$ & 89.8 & .134 & 4 & Fin & 6.8 & -.009 \\
\hline $1960.5440^{\diamond *}$ & 88.2 & .12 & - & $V B S$ & 5.7 & -.021 \\
\hline $1960.6400^{*}$ & 84.5 & .127 & 1 & Fin & 1.8 & -.014 \\
\hline $1961.5900^{\diamond *}$ & 89.0 & .13 & - & $B$ & 6.8 & -.007 \\
\hline $1962.6400^{*}$ & 97.9 & .129 & 5 & Fin & 15.8 & .000 \\
\hline 1963.6350 & - & $<.122$ & 1 & Fin & (261.4) & $(.121)$ \\
\hline $1963.6380^{* *}$ & $: 133$. & $: .111$ & 1 & Fin & 128.5 & -.010 \\
\hline 1964.6540 & - & $<.114$ & 1 & Fin & $(260.9)$ & $(.100)$ \\
\hline 1965.6350 & - & $<.104$ & 1 & Fin & $(260.4)$ & $(.097)$ \\
\hline 1966.6430 & - & $<.102$ & 1 & Fin & (259.8) & $(.083)$ \\
\hline 1976.2963 & 83.1 & .080 & 1 & $M c A$ & -2.8 & -.002 \\
\hline 1976.3699 & 85.2 & .088 & 1 & $M c A$ & -.7 & .005 \\
\hline 1976.4573 & 83.7 & .089 & 1 & $M c A$ & -2.1 & .005 \\
\hline 1977.3338 & 82.8 & .097 & 1 & $M c A$ & -2.4 & .001 \\
\hline 1977.4813 & 83.3 & .097 & 1 & $M c A$ & -1.8 & -.001 \\
\hline 1978.1501 & 85.8 & .101 & 1 & $M c A$ & 1.0 & -.006 \\
\hline 1978.6173 & 83.9 & .108 & 1 & $M c A$ & -.6 & -.004 \\
\hline 1979.5291 & 83.5 & .112 & 1 & $M c A$ & -.7 & -.009 \\
\hline 1980.1597 & 85.0 & .120 & 1 & $M c A$ & 1.0 & -.007 \\
\hline 1980.4766 & 83.7 & .124 & 1 & $M c A$ & -.1 & -.005 \\
\hline 1980.7225 & 81.1 & .110 & 1 & $M c A$ & -2.7 & -.021 \\
\hline 1981.4568 & 84.1 & .119 & 1 & $M c A$ & .6 & -.016 \\
\hline 1981.4677 & 83.4 & .119 & 1 & $M c A$ & -.1 & -.016 \\
\hline 1981.4731 & 83.5 & .119 & 1 & $M c A$ & .0 & -.016 \\
\hline 1983.0702 & 84.9 & .121 & 1 & $H r t$ & 1.8 & -.019 \\
\hline 1983.4200 & 82.9 & .125 & 1 & $M c A$ & -.1 & -.015 \\
\hline 1983.4282 & 82.6 & .130 & 1 & $H r t$ & -.4 & -.010 \\
\hline 1983.7151 & 85.6 & .127 & 1 & $M c A$ & 2.7 & -.013 \\
\hline 1984.3730 & 83.7 & .132 & 1 & $M c A$ & 1.0 & -.008 \\
\hline 1984.3757 & 84.0 & .126 & 1 & $M c A$ & 1.3 & -.014 \\
\hline 1985.4843 & 84.1 & .126 & 1 & $M c A$ & 1.7 & -.010 \\
\hline $1985.5100^{\circ}$ & 77.4 & .13 & - & $\mathrm{Cou}$ & -4.9 & -.006 \\
\hline 1986.4098 & 84.0 & .127 & 1 & $M c A$ & 1.8 & -.003 \\
\hline 1987.2726 & 84.9 & .124 & 1 & $M c A$ & 3.1 & .000 \\
\hline 1988.2501 & 83.3 & .122 & 1 & $M c A$ & 1.9 & .008 \\
\hline 1988.2527 & 83.2 & .118 & 1 & $M c A$ & 1.8 & .004 \\
\hline 1989.2275 & 84.1 & .115 & 1 & $M c A$ & 3.2 & .013 \\
\hline 1990.2625 & 83.5 & .112 & 1 & $H r t$ & 3.2 & .024 \\
\hline 1991.2500 & 101. & .125 & 1 & Hip & 21.6 & .053 \\
\hline 1991.3191 & 79.7 & .102 & 1 & $\mathrm{Hrt}$ & .3 & .031 \\
\hline 1991.7258 & 78.2 & .089 & 1 & $H r t$ & -0.6 & .024 \\
\hline 1992.3075 & 79.3 & .087 & 1 & $H r t$ & 1.4 & .032 \\
\hline
\end{tabular}


D. Olević and Z. Cvetković: Orbits of 10 interferometric binary systems calculated by using Koval'skij method, Online Material p 4

Table 2. continued.

\begin{tabular}{crrllrr}
\hline \hline \multicolumn{7}{c}{ WDS $17018-5108=$ I 1306} \\
\hline$t$ & $\theta$ & $\rho$ & $n$ & Obs. & $\Delta \theta$ & $\Delta \rho$ \\
\hline $1926.5100^{\circ *}$ & 199.9 & .36 & - & $I$ & 9.9 & .144 \\
$1929.3200^{\diamond *}$ & 196.2 & .28 & - & Vou & 4.5 & .047 \\
$1952.4800^{\circ}$ & 202.2 & .20 & - & B & -0.4 & .062 \\
1953.6200 & 202.9 & .141 & 5 & Fin & -1.4 & -.003 \\
1953.6600 & 209.2 & .143 & 1 & Fin & 4.8 & -.002 \\
1954.6100 & 206.8 & .136 & 2 & Fin & 1.0 & -.009 \\
1959.6900 & 215.0 & .114 & 4 & Fin & 0.1 & .000 \\
1960.6900 & - & $<.113$ & 1 & Fin & $(217.1)$ & $(.105)$ \\
1960.7000 & - & $<.105$ & 1 & Fin & $(217.2)$ & $(.105)$ \\
1962.6800 & - & $<.108$ & 1 & Fin & $(224.0)$ & $(.086)$ \\
1963.6630 & - & $<.109$ & 1 & Fin & $(228.8)$ & $(.076)$ \\
1964.6980 & - & $<.107$ & 1 & Fin & $(235.2)$ & $(.066)$ \\
$1985.3300^{\circ *}$ & 191.8 & .20 & - & hz & 4.9 & .011 \\
1989.3038 & 10.6 & .219 & 1 & McA & 0.8 & .000 \\
1990.3496 & 10.8 & .225 & 1 & Hrt & 0.4 & -.001 \\
$1991.2500^{*}$ & 190.0 & .231 & 1 & Hip & -0.9 & .000 \\
1992.4550 & 11.4 & .239 & 1 & Hrt & -0.2 & .002 \\
\hline
\end{tabular}

Table 2. continued.

\begin{tabular}{|c|c|c|c|c|c|c|}
\hline \multicolumn{7}{|c|}{ WDS 17221-7007 = FIN 373} \\
\hline$t$ & $\theta$ & $\rho$ & $n$ & Obs. & $\Delta \theta$ & $\Delta \rho$ \\
\hline 1960.6900 & 119.2 & .110 & 3 & Fin & -0.3 & .001 \\
\hline 1962.6800 & 125.8 & .120 & 4 & Fin & 0.1 & -.001 \\
\hline 1963.6720 & 135.2 & .114 & 4 & Fin & 6.7 & -.009 \\
\hline 1964.7000 & 124.8 & .119 & 3 & Fin & -6.5 & -.003 \\
\hline 1966.7120 & 139.2 & .121 & 3 & Fin & 2.0 & .004 \\
\hline 1989.3066 & 280.9 & .104 & 1 & $M c A$ & 5.3 & .005 \\
\hline 1991.2500* & 100.0 & .091 & 1 & Hip & -4.2 & -.002 \\
\hline 1992.4496 & 290.1 & .077 & 1 & $H r t$ & -0.9 & .000 \\
\hline
\end{tabular}

